ARTICLE

https://doi.org/10.1038/s41467-019-13627-y

\title{
Metabolic balancing by miR-276 shapes the mosquito reproductive cycle and Plasmodium falciparum development
}

\author{
Lena Lampe ${ }^{1,3}$, Marius Jentzsch $^{1}$, Sylwia Kierszniowska² \& Elena A. Levashina (i) ${ }^{1 \star}$
}

The blood-feeding behavior of Anopheles females delivers essential nutrients for egg development and drives parasite transmission between humans. Plasmodium growth is adapted to the vector reproductive cycle, but how changes in the reproductive cycle impact parasite development remains unclear. Here, we show that the bloodmeal-induced miR-276-5p fine-tunes the expression of branched-chain amino acid transferase to terminate the reproductive cycle. Silencing of miR-276 prolongs high rates of amino acid (AA) catabolism and increases female fertility, suggesting that timely termination of $A A$ catabolism restricts mosquito investment into reproduction. Prolongation of $A A$ catabolism in $P$. falciparuminfected females also compromises the development of the transmissible sporozoite forms. Our results suggest that Plasmodium sporogony exploits the surplus mosquito resources available after reproductive investment and demonstrate the crucial role of the mosquito AA metabolism in within-vector parasite proliferation and malaria transmission.

\footnotetext{
${ }^{1}$ Vector Biology Unit, Max Planck Institute for Infection Biology, Charitéplatz 1, 10117 Berlin, Germany. ${ }^{2}$ metaSysX GmbH, 14476 Potsdam-Golm, Germany.

${ }^{3}$ Present address: Physiology and Metabolism Laboratory, Francis Crick Institute, 1 Midland Road, NW1 1AT London, UK. *email: levashina@mpiib-berlin.mpg.de
} 
$\mathrm{T}$ he hematophagous lifestyle of Anopheles females is an efficient reproductive strategy that is exploited by the malaria parasite for its transmission. Mosquitoes mostly feed on carbohydrate-rich plant nectars and only females take blood to kick-start their reproductive cycle. The blood meal provides the female with a nutritional boost in amino acids and lipids that is crucial for egg development. This extreme change in diet triggers massive coordinated metabolic changes in multiple mosquito tissues to ensure robust egg development within a 3-day time window.

The insect fat body is the main nutrient storage organ and plays a central role in the mosquito metabolism. During a pre-bloodfeeding anabolic phase, the fat body accumulates nutrients in the form of triacylglycerides (TAGs) and glycogen derived from plant nectars ${ }^{1-4}$. A successful blood feeding massively increases levels of free amino acids (AAs) in the mosquito circulation, that are sensed in the fat body by the target of rapamycin (TOR) pathway ${ }^{5-7}$. Activation of TOR, together with the increasing levels of the steroid hormone 20-hydroxyecdysone (20E), initiate a switch from anabolic to catabolic metabolism ${ }^{1,2,7}$. In the fat body, blood mealderived AAs feed into the tricarboxylic acid (TCA) cycle to generate energy for nutrient mobilization and are incorporated into major yolk proteins such as lipophorin and vitellogenin ${ }^{8,9}$. Inhibition of nutrient transport, metabolism, or the TOR signaling pathway dramatically reduces egg production ${ }^{5,10-13}$. The mosquito reproductive cycle can be roughly separated into three phases ${ }^{1,2}$ : During the early ( $0-10 \mathrm{~h}$ post blood feeding $(\mathrm{hpb}))$ and mid-phase $(10-36 \mathrm{hpb})$, the metabolic program prioritizes the mobilization of nutrients for rapid egg development. During the late phase (36-72 hpb), production of the yolk proteins in the fat body ceases, and transcriptional and metabolic programs return to the anabolic state to replenish consumed glycogen and lipid reserves. The metabolic switch from catabolism to anabolism during the late phase prepares females for the next reproductive cycle. Indeed, prolongation of the reproductive cycle by inhibition of autophagy or silencing of a negative regulator of TOR signaling in the fat body curbs egg production in the second reproductive cycle ${ }^{14,15}$. Therefore, both the induction and termination of the reproductive cycle are essential for female fertility.

Mosquitoes acquire malaria parasites when feeding on blood infected with $P$. falciparum sexual stages that fuse in the mosquito midgut to produce motile ookinetes. The ookinetes traverse the midgut epithelium at $18-24 \mathrm{hpb}$ and transform into oocysts to establish infection at the basal side of the midgut wall. Here, the parasites undergo massive replication and maturation (also called sporogony). Within two weeks of sporogony, the parasite biomass increases dramatically generating up to 1000 sporozoites within each oocyst ${ }^{16}$. Developing oocysts require large amounts of nutrients, such as AAs, sugars, and lipids, for which they rely on the mosquito environment. Therefore, it is not surprising that the mosquito's nutritional status largely correlates with parasite development ${ }^{17,18}$. Inhibition of sugar uptake or lipid access of the Plasmodium oocysts decreases the number and virulence of transmissible sporozoites ${ }^{19,20}$. As sporogony occurs after completion of the mosquito reproductive cycle, the oocysts do not directly compete with the vector but rely on unconsumed resources stored in the mosquito tissues ${ }^{19,21}$.

Hormonal signals, in particular 20E, orchestrate the transcriptional and post-transcriptional networks that shape the mosquito post-blood meal metabolism ${ }^{1,2,7}$. Indeed, 20E-driven metabolic changes are crucial for release of stored and blood meal-derived nutrients for mosquito reproduction as well as parasite development $^{21}$. MicroRNAs contribute to post-transcriptional regulation and link the endocrine regulation with metabolic homeostasis in Aedes mosquitoes ${ }^{22-24}$. Using a transcriptomic approach in A. gambiae, we identified three miRNAs whose fat body expression was induced shortly after blood feeding, namely miR-275,
miR-276, and miR-305 ${ }^{25}$. Consistently with our findings, miR-275 was reported to regulate blood meal digestion and egg development $^{24}$, while miR-305 was shown to impact mosquito microbiota and $P$. falciparum development by an as yet unknown mechanism $^{26}$. Here, we report the role of miR-276 in fine-tuning the expression of branched chain amino acid transferase (BCAT) in the fat body. We show that miR-276 depletion prolongs high levels of $B C A T$ expression and AA catabolism in the fat body, thereby increasing female fertility. Unexpectedly, sustained high AA catabolism compromises $P$. falciparum sporogonic development and reduces the number of transmissible sporozoites. Our results demonstrate the important role of mosquito metabolism in vector competence and malaria transmission.

\section{Results}

AAs and ecdysone regulate miR-276 in the fat body. To define the role of miR-276 in the mosquito reproductive cycle, we examined its expressional profile by reverse transcription quantitative real-time PCR (RT-qPCR) in the abdominal carcass tissues collected before and after blood feeding. As the fat body is highly enriched in abdominal carcasses, we refer to them as fat body samples hereafter. Newly-eclosed females showed high levels of miR-276 expression that declined on the second day after eclosion (Supplementary Fig. 1). A blood meal transiently increased miR-276 levels from 28 to $44 \mathrm{hpb}$ (Fig. 1a). This expression pattern paralleled the kinetics of the steroid hormone ecdysone, a key regulator of mosquito development and reproduction, whose synthesis is triggered by a blood meal. To compare the timing of miR-276 expression and ecdysone titers, we measured the levels of the metabolically active form of ecdysone, 20-hydroxyecdysone (20E), in the blood-fed females by an enzyme-linked immunosorbent assay (ELISA) (Fig. 1a). 20E titers preceded the induction of miR-276 expression as they increased from 6 to $24 \mathrm{hpb}$ and fell down to basal levels at $36 \mathrm{hpb}$. Based on these results, we hypothesized that expression of miR-276 in the fat body may be regulated by $20 \mathrm{E}$.

As both $20 \mathrm{E}$ and AAs regulate the mosquito reproductive cycle, we gauged expression levels of miR-276 after stimulation with $20 \mathrm{E}$ or/and AAs in an ex vivo fat body culture system ${ }^{27,28}$. The abdominal carcasses were incubated in culture medium supplemented or not with $20 \mathrm{E}$ or/and AAs in vitro. Addition of AAs or $20 \mathrm{E}$ increased miR-276 levels by two-fold. Moreover, a significant five-fold induction of miR-276 expression was observed when AA and $20 \mathrm{E}$ were added together (Fig. $1 \mathrm{~b}$ ). These results suggested a synergistic effect of AA and $20 \mathrm{E}$ on regulation of miR-276 expression in the fat body after a blood meal. In line with these results, bioinformatics analysis identified one potential binding site for the ecdysone-induced transcriptional factor Broad in the upstream regulatory region of the miR-276 gene.

miR-276 inhibits the branched chain amino acid transferase. MicroRNAs post-transcriptionally promote transcript degradation or inhibition of translation by binding to specific sites located predominantly in the $3^{\prime} \mathrm{UTR}$. To identify putative miR276 mRNA targets, we applied three in silico target prediction tools, namely MiRanda ${ }^{29}$, RNAhybrid ${ }^{30}$ and MicroTar ${ }^{31}$ (Supplementary Data 1 ). We next used a publicly available RNAseq dataset $^{7}$ to identify the fat body-expressed candidate target mRNAs with the reversed expression pattern to miR-276. This dual approach allowed us to down-select eight putative targets for further analyses: ATP synthase (AGAP012081), branched chain amino acid transferase (BCAT; AGAP000011), glycerol-3phosphate dehydrogenase (GPD; AGAP004437), methylmalonatesemialdehyde dehydrogenase (MMSDH; AGAP002499), NADH dehydrogenase (NADH (I), AGAP010464), 3-methylcrotonyl-CoA 
a

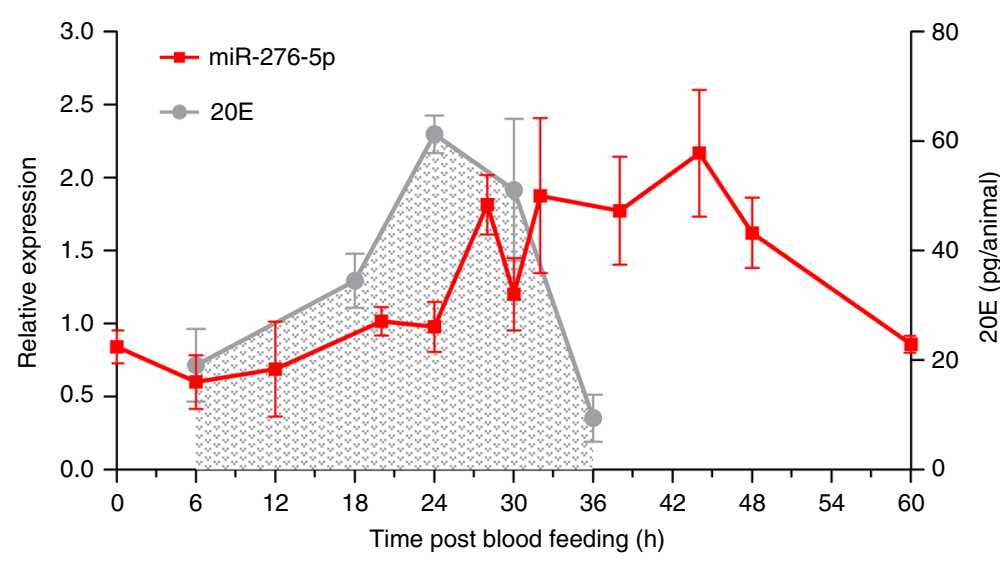

b

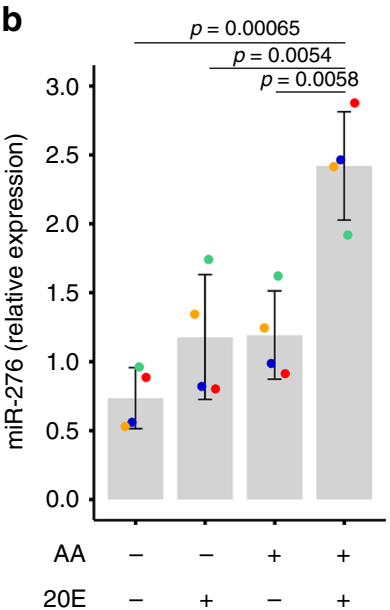

Fig. 1 Amino acid (AA) and steroid hormone (20E) signalling regulate miR-276 expression. a miR-276 (red) expression in the female fat body ( $n=10$, $N=6)$ and 20-hydroxyecdysone (20E) titres (gray) in the mosquito females $(n=9, N=3)$ at different time points after blood feeding. The plots show mean \pm SEM of independent experiments. $\mathbf{b}$ miR-276 expression in ex vivo fat body cultures of 3-4-day-old females $(n=3, N=4)$ after incubation with medium with or without amino acids (AA) and 20E. miRNA expression levels were normalized using the ribosomal protein RPS7 gene. Boxplots show the median with first and third quartile, whiskers depict the min and max of independent experiments, colored dots show mean of each experiment. The statistical significance was tested by one-way ANOVA followed by Tukey's post hoc test and the obtained $p$-values are shown above the horizontal lines ( $n=$ number of mosquitoes pooled for each independent experiment; $N=$ number of independent experiments).

carboxylase (MCC; AGAP010228), branched-chain alpha-keto acid dehydrogenase (BCKDH, AGAP003136) and mitochondrial ornithine receptor (MOR; AGAP000448).

We postulated that expression of these target transcripts should be repressed by miR-276 and measured transcript levels of the selected mRNAs in the fat bodies of miR-276 knockdown mosquitoes (miR-276 ${ }^{\mathrm{KD}}$ ) at the peak of miR-276 expression (38 $\mathrm{hpb}$ ) by RT-qPCR. miR-276 was silenced by injection of antisense oligonucleotides (miR-276 antagomirs), whereas injection of a scrambled antagomir served as control (Supplementary Table 2). Out of the examined candidates, only expression of $B C A T$ significantly increased after miR-276 inhibition (Fig. 2a). BCAT carries one predicted miR-276 binding site in its $3^{\prime} \mathrm{UTR}$ (Fig. 2b). However, as we did not perform genome-wide analysis of mRNA changes following miR-276 inhibition, we cannot exclude that miR-276 regulates other mRNAs than BCAT.

We next tested for direct interaction between miR-276 and the binding site in the BCAT $3^{\prime}$ UTR using a Dual Luciferase Reporter Assay in the Drosophila Schneider 2 (S2) cell cultures in vitro. This assay examines the ability of miR-276 to posttranscriptionally inhibit a reporter construct containing the Renilla luciferase reporter gene fused to the BCAT 3'UTR. We generated three constructs: (1) a positive control, which carries three copies of the miR-276 binding site ( $3 \mathrm{x}$ binding site), (2) the wild-type $B C A T 3^{\prime} \mathrm{UTR}$ with an intact single miR-276 binding site (BCAT 3'UTR); and (3) a BCAT 3'UTR negative control with the mutated sequence of miR-276 binding site (BCAT $3^{\prime} \mathrm{UTR}^{\mathrm{mut}}{ }^{\text {). }}$. S2 cells expressed low levels of endogenous Drosophila miR-276 leading to basal inhibition of the BCAT 3'UTR reporter activity. Co-transfection of the mosquito miR-276 further enhanced inhibition of the luciferase reporter fused to the functional 3'UTR binding sites but not to the mutated control (Supplementary Fig. 2). Importantly, mosquito miR-276 significantly repressed expression of the positive control reporter with the three copies of the miRNA binding site and the wild-type BCAT $3^{\prime} \mathrm{UTR}$ reporter. In contrast, mutations in the BCAT miRNA binding site abolished the inhibition of the luciferase reporter by more than two-fold (Fig. 2c and Supplementary Fig. 2). These results demonstrated that BCAT $3^{\prime} \mathrm{UTR}$ is a bona fide target of miR-276.
To confirm our in vitro findings, we examined $B C A T$ transcript levels in vivo after blood feeding in miR-276 $\mathrm{KD}$ and scrambleinjected mosquitoes. We observed that in the presence of miR276, BCAT transcript levels went down as early as $24 \mathrm{hpb}$, inhibition of the miRNA function by antagomir prolonged $B C A T$ expression by almost $12 \mathrm{~h}$ (Fig. $2 \mathrm{~d}$ ). We concluded that miR-276 tunes down BCAT expression during the late phase of the mosquito reproductive cycle.

miR-276 fine-tunes AA metabolism of the reproductive cycle. BCAT catalyzes the first step of the branched chain amino acid (leucine, valine, and isoleucine) catabolism. As miR-276 posttranscriptionally repressed $B C A T$ during the late phase of the reproductive cycle, we examined the amino acid metabolism after miR-276 knockdown. We compared metabolite profiles of miR$276^{\mathrm{KD}}$ and control females (whole mosquitoes) at three time points after $P$. falciparum-infected blood feeding (10, 38, and 48 hpb) using gas chromatography-mass spectrometry (GC-MS) and liquid chromatography-mass spectrometry (LC-MS) (Supplementary Data 1$)$. We selected time points before $(10 \mathrm{hpb})$ and during miR-276 expression (38 and $48 \mathrm{hpb}$ ). In total, we detected 4,716 chromatographic peaks $(\mathrm{m} / z$ at a specific retention time) of which 816 were annotated in reference of authentic standards. The annotated data were normalized to the sample median and filtered using the interquartile range. The data were further $\log _{2}$-transformed and pareto scaled for heatmap visualization. 294 features significantly changed across the reproductive cycle (two-way ANOVA; Supplementary Data 2) and were included in the heatmap. Massive metabolic changes after blood feeding were observed in both control and miR-276 ${ }^{\mathrm{KD}}$ mosquitoes (Fig. 3a). As early as $10 \mathrm{hpb}$, a large cluster of highly enriched metabolites correlated with the influx of human blood (Cluster I). This cluster comprised amino acids, short and long chain fatty acids, as well as sphingomyelins, potentially representing metabolites derived from the ingested blood. A second cluster (Cluster II), comprising predominantly dipeptides, was abundant at 10 and $38 \mathrm{hpb}$ and disappeared at the late phase of the reproductive cycle (48 hpb). Conversely, Cluster III featured the metabolites whose levels increased at 38 and $48 \mathrm{hpb}$, corresponding to the periods of 
a

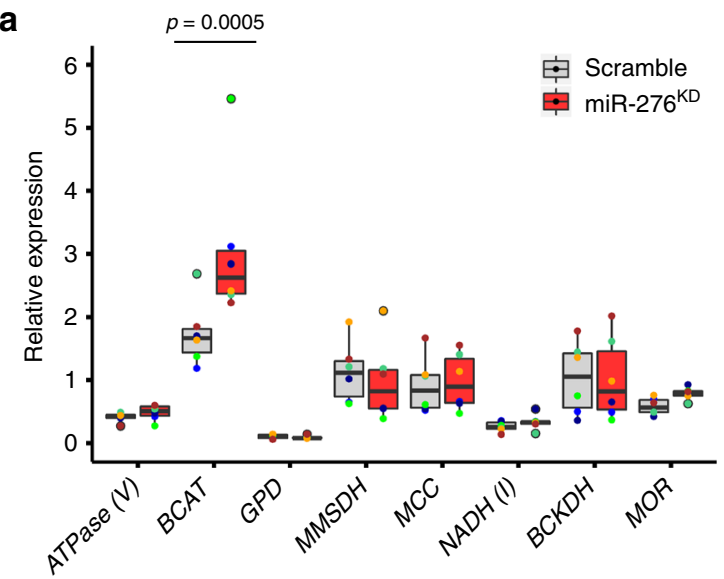

b

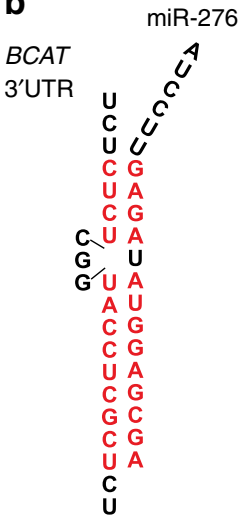

C

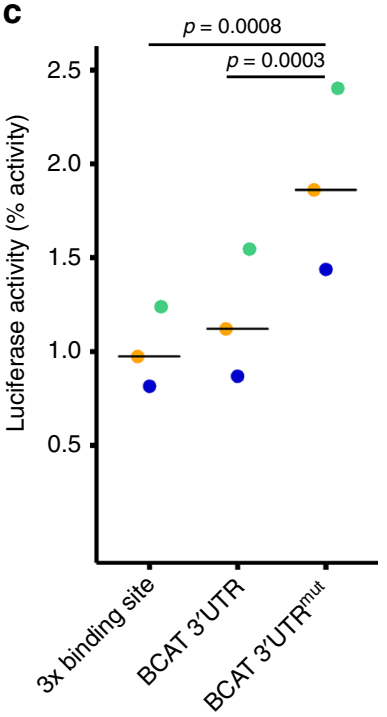

d

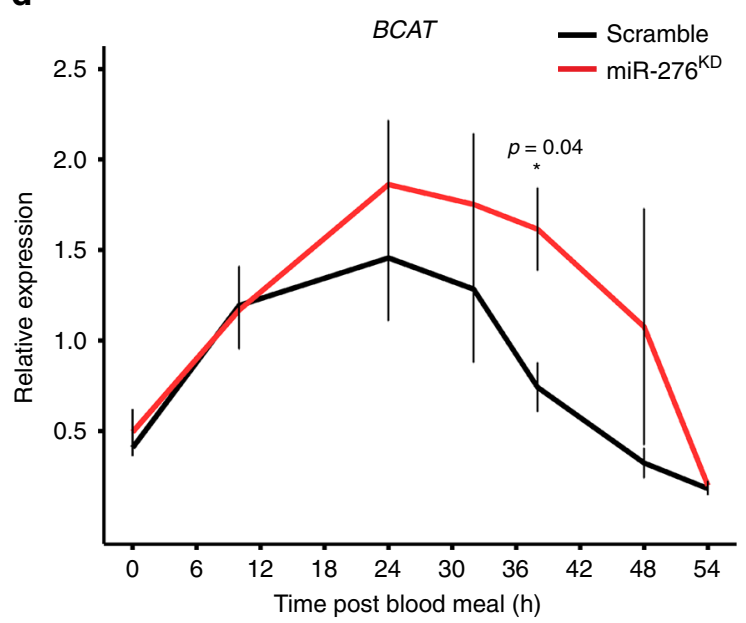

Fig. 2 miR-276 post-transcriptionally represses the branched-chain amino acid transferase in the mosquito fat body. a Expression of predicted miRNA targets at $38 \mathrm{~h}$ post blood feeding in the fat body of mosquitoes $(n=5, N=6)$ injected with anti-miR-276 (miR-276, red) or scrambled antagomir (scramble, gray). Expression of the ATP synthase (ATPase V), branched chain amino acid transferase (BCAT), glycerol-3-phosphate dehydrogenase (GPD), methylmalonate-semialdehyde dehydrogenase (MMSDH), 3-methylcrotonyl-CoA carboxylase (MCC), NADH dehydrogenase (NADH (I)), branched-chain alphaketo acid dehydrogenase (BCKDH) and mitochondrial ornithine receptor (MOR). Expression levels were normalized using the ribosomal protein RPS7 gene. Boxplots show the median with first and third quartile, whiskers depict the min and max. Statistical significance was tested by two-way ANOVA followed by Tukey's post hoc test and significant differences are shown by the $p$-value above the horizontal line. $\mathbf{b}$ Sequence of the predicted miR-276 binding site in the BCAT 3'UTR. Red color indicates complementary nucleotides in the $3^{\prime} U T R$ binding site and miR-276. c Dual luciferase reporter assay in vitro. A construct containing three copies of miR-276 binding sites served as positive control. Lines depict medians and colored dots show means of independent experiments $(N=3)$. Statistical significance of differences was tested by one-way ANOVA followed by Tukey's post hoc test and significant differences are indicated by the $p$-values above the horizontal lines. $\mathbf{d} B C A T$ expression in the fat body during the reproductive cycle of mosquitoes injected with anti-miR276 (miR-276 KD, red) or scrambled antagomir (scramble, black). Expression levels were normalized using the ribosomal protein RPS7 gene. Means \pm SEM (vertical lines) were plotted. Statistically significant differences between miR-276 KD and control mosquitoes were determined by two-way ANOVA followed by Tukey's post hoc test $(N=3)$ and significant differences are shown by the $p$-value $\left.{ }^{*}\right)(n=$ number of mosquitoes pooled for each independent experiment; $N=$ number of independent experiments).

complete blood digestion and ovary development. The vast majority of Cluster III metabolites were lipids (triacylglcerides (TAGs), phosphatidiylcholines, phosphatidiylethanolamines, phosphatidiylinositols), and trehalose (Fig. 3a). Overall, the observed metabolite dynamics were not significantly perturbed by miR-276 $\mathrm{KD}$ and closely recapitulated the major physiological processes induced by blood feeding. This observation was in line with the fine-tuning role of miR-276, whose silencing did not hinder the overall blood feeding-induced metabolic profile.

We next focused on BCAT-related metabolites. BCAT initiates BCAA catabolism by transferring the BCAA amino group to a-ketoglutarate forming glutamate, which is thought to serve as a nitrogen sink in insects ${ }^{32}$ (Fig. 3b). While BCAA levels did not massively change in $\mathrm{miR}-276^{\mathrm{KD}}$ mosquitoes, significantly higher levels of glutamate were detected at $48 \mathrm{hpb}$ (Fig. 3c). Furthermore, miR-276 $\mathrm{KD}$ also increased levels of histidine, lysine, citrulline, arginine, argininosuccinate and ornithine at $48 \mathrm{hpb}$ (Fig. 3d), pointing to an accumulation of metabolites associated with nitrogen metabolism, the waste product of AA catabolism, during the late phase of reproductive cycle. The detected increase in nitrogen metabolism in miR- $276^{\mathrm{KD}}$ mosquitoes during the late phase of the reproductive cycle $(48 \mathrm{hpb})$ is consistent with the transcriptional increase in BCAT levels observed in miR-276 $\mathrm{KD}$ females. Taking together, our results suggest that miR-276 
a

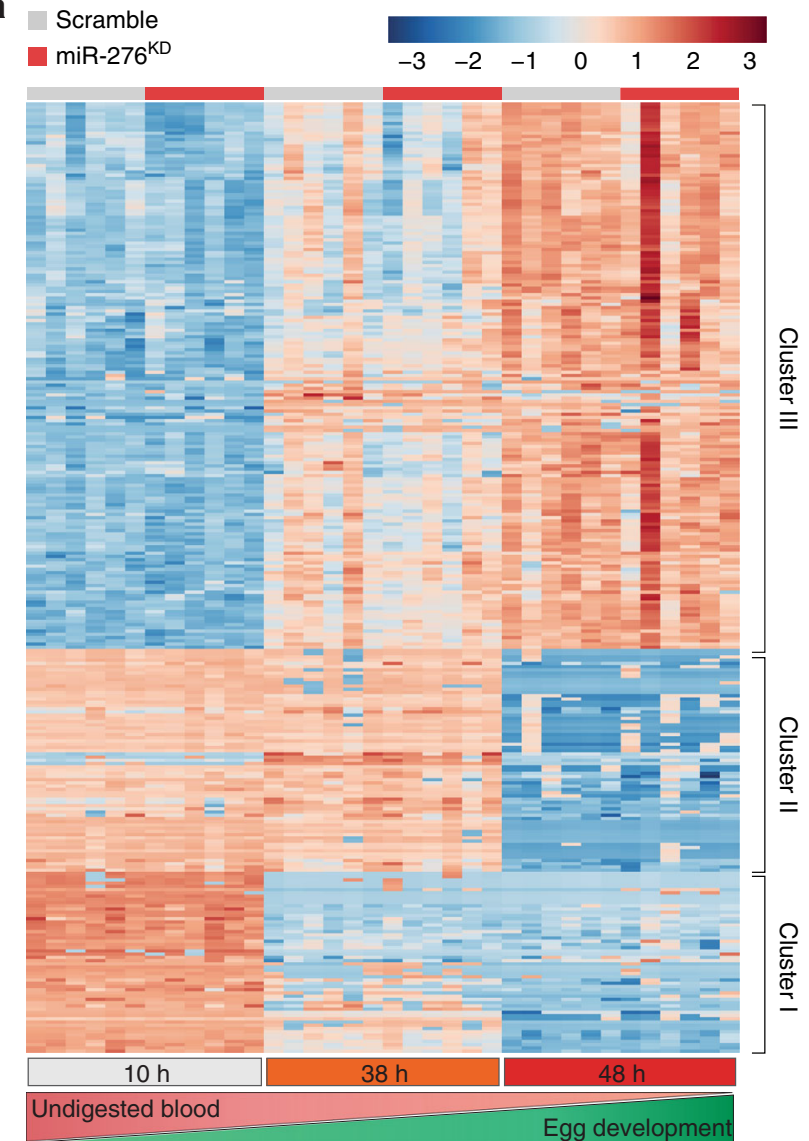

d

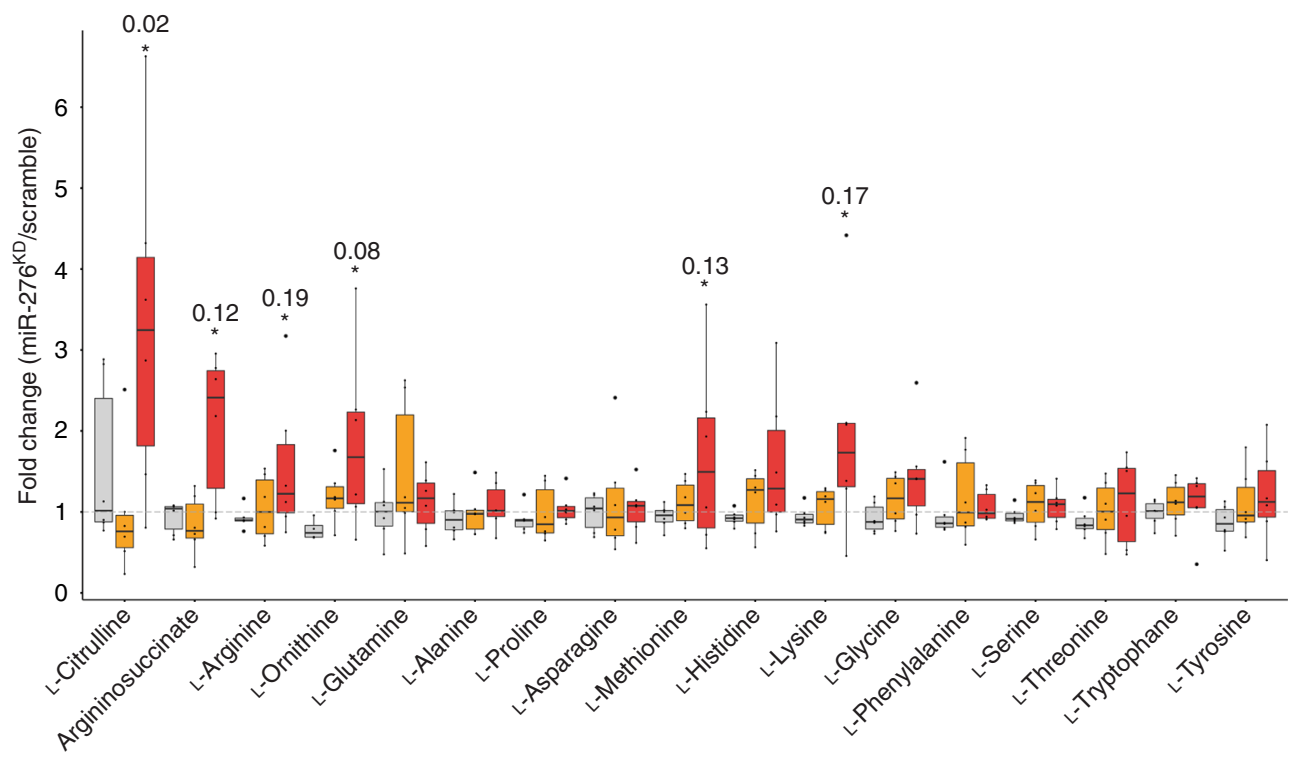

b

BCAAs

(Leu, Val, Ile)

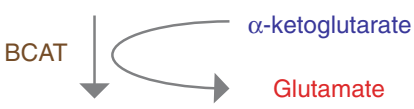

$\alpha$-ketoacids

$+$

TCA cycle
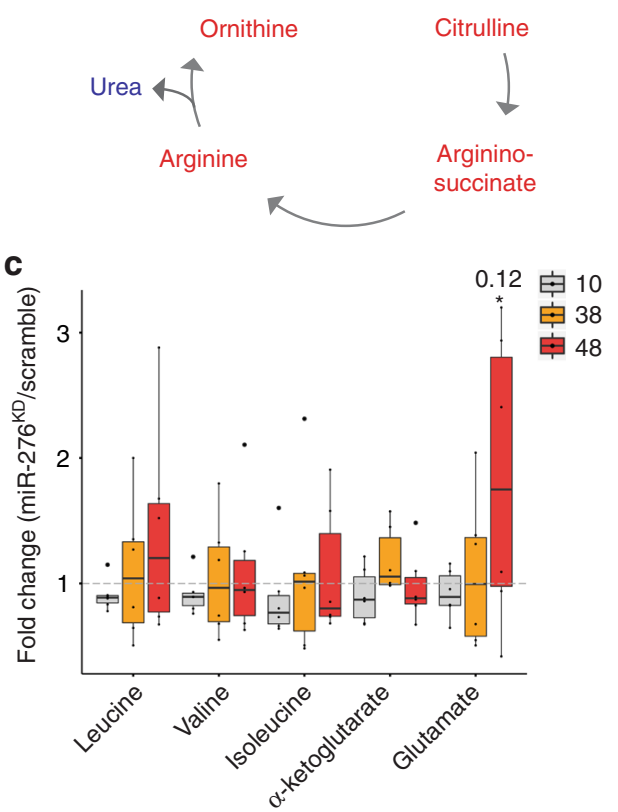

contributes to the termination of the AA catabolism by finetuning BCAT transcript levels and that miR-276 silencing prolongs the catabolic phase of the mosquito reproductive cycle.

miR-276 regulates oogenesis and $P$. falciparum sporogony. Both mosquitoes and malaria parasites rely on nutrients for successful ovary and sporogonic development, respectively. We first examined how prolongation of the catabolic phase in miR-276 ${ }^{\mathrm{KD}}$ mosquitoes affects oogenesis. Female mosquitoes were injected with the miR-276 or control (scramble) and blood fed three days later to induce egg development. Two days later, females were transferred into egg laying chambers and the numbers of eggs laid by individual females were enumerated. Inhibition of miR-276 significantly increased egg laying (fertility) without compromising larval hatching rates (fecundity) (Fig. 4a and Supplementary Fig. 5). To provide further evidence that $B C A T$ is the target of miR-276, we examined the effect of BCAT knockdown on oogenesis. In line with the results obtained for 
Fig. 3 miR-276 fine-tunes amino acid (AA) metabolism in the late phase of the mosquito reproductive cycle. a Heatmap of annotated metabolite levels that vary across the reproductive cycle. Metabolite levels were measured by GC-MS and LC-MS from whole females $(n=10, N=6)$ at 10,38 , and $48 \mathrm{~h}$ post $P$. falciparum-infected blood meal in control (scrambled, gray) and miR-276-depleted (miR-276 KD, red) mosquitoes. Bottom bars indicate time points after blood feeding ( $10 \mathrm{~h}$, gray; $38 \mathrm{~h}$, orange; $48 \mathrm{~h}$, red), and gradients below show the progression of blood digestion (red) and ovary development (green). Samples are plotted on the $x$-axis and metabolites on the $y$-axis. For heatmap visualization, data were $\log _{2}$ transformed and pareto scaled. $\mathbf{b}$ Overview of the classical enzymatic activity of the branched chain aminotransferase (BCAT) in animals. The amino group of BCAAs is transferred to $\alpha$-ketoglutarate by BCAT resulting in glutamate. Glutamate serves as a nitrogen sink, which is passed on to the urea cycle in vertebrates. As mosquitoes lack the carbamoyl phosphate synthase, the link between glutamate and citrulline of the urea cycle is unknown. Within the classical urea cycle, citrulline is converted to argininosuccinate and subsequently arginine, which is metabolized to ornithine ultimately releasing urea. Metabolite changes upon miR-276 inhibition $(48 \mathrm{hpb})$ are shown in red, unchanged metabolites are in blue, and undetected metabolites are in gray. c Fold-change differences in branched chain amino acids (leucine, valine, isoleucine), $\alpha$-ketoglutarate and glutamate detected in miR-276 $\mathrm{KD}$ as compared to scramble controls. $\mathbf{d}$ Fold change differences in metabolites of AA metabolism at $10 \mathrm{~h}$ (gray), $38 \mathrm{~h}$ (orange), $48 \mathrm{~h}$ (red) post blood feeding in whole females. Statistical significance of differences in metabolite levels of miR-276 KD and scramble controls was tested by $t$-test $(n=$ number of mosquitoes pooled for each independent experiment; $N=$ number of independent experiments).
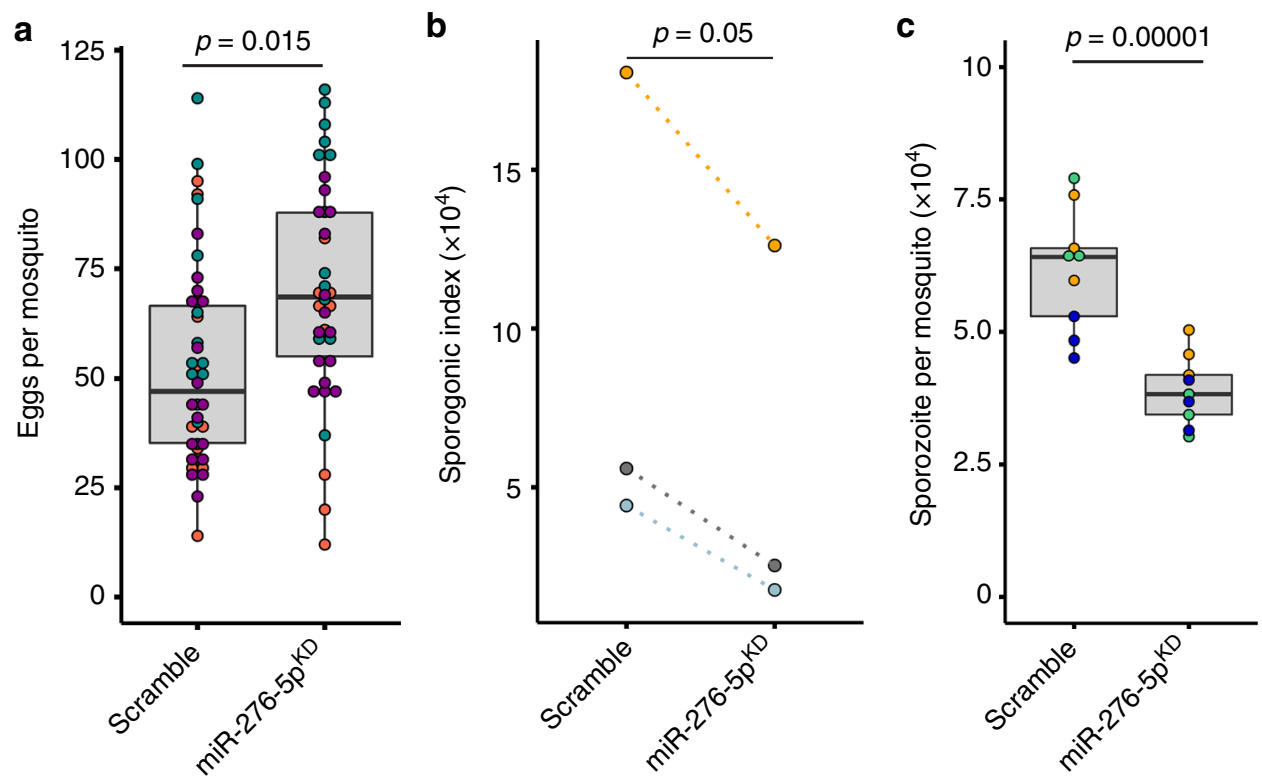

Fig. 4 Effects of miR-276 silencing on mosquito fertility and $\boldsymbol{P}$. falciparum sporogonic development. a Egg laying rates (fertility) of individual females injected with miR-276 (miR-276 KD) or control antagomir (scramble) after a blood meal. Boxplots show the median with first and third quartile, whiskers depict min and max values. Each dot represents one mosquito and dot colors indicate independent experiments ( $N=3$, Supplementary Table 3 ). b Females injected with miR-276 (miR-276 KD) or control (scramble) antagomir were infected with P. falciparum. At day 11 post infection, oocyst number and size were measured. The median of $P$. falciparum oocysts per midgut and mean of $P$. falciparum oocyst size per experiment were multiplied to generate a sporogonic index $(N=3$, Supplementary Table 3). c The numbers of the salivary gland sporozoites per mosquito were quantified on day 14 post infection. Boxplots show the median with first and third quartile, whiskers depict min and max values. Colors show replicates of each independent experiment. Statistically significant differences are indicated by $p$-values above the horizontal lines deduced by one-way ANOVA and Tukey's post-hoc test $(N=3$, Supplementary Table 3), $N=$ number of independent experiments.

miR-276 ${ }^{\mathrm{KD}}, B C A T$ silencing by injection of dsRNA prior to blood feeding decreased egg laying, suggesting a critical role of amino acid catabolism in mosquito reproduction (Supplementary fig. 6). We concluded that miR-276-BCAT axis regulates mosquito metabolic investment into oogenesis by terminating the catabolic phase before complete nutrient consumption by oogenesis.

We next asked whether the observed shift in the metabolic investment into oogenesis impacts parasite development. Using the same experimental settings, we infected miR-276 $\mathrm{KD}$ and control mosquitoes with $P$. falciparum and gauged the number and size of oocysts 11 days post infection. Similarly to previous reports, wounding by injection of scramble antagomir decreased parasite infection load ${ }^{33}$ (Supplementary Fig. 4). Importantly, inhibition of miR-276 further significantly reduced either the median number or the mean size of $P$. falciparum oocysts. To quantify oocyst development, we calculated a sporogonic index by multiplying the median number by the mean size of oocysts per midgut in each independent experiment. We observed that regardless of infection levels, the sporogonic index was significantly lower in miR-276 KD mosquitoes than in controls (Fig. 4b). We further examined whether the observed decrease in the parasite mass translated into lower numbers of the salivary gland sporozoites. Indeed, miR-276 inhibition significantly reduced by two-fold the loads of the salivary gland sporozoites (Fig. 4c), suggesting that prolongation of the catabolic phase restricts resources available for the development of the parasite transmissible forms.

In summary, our results demonstrated that the miR-276regulated switch from catabolic to anabolic amino acid metabolism in the fat body restricts mosquito investment into oogenesis and benefits $P$. falciparum development. 


\section{Discussion}

The hematophagous lifestyle endows mosquitoes with a speedy within-days egg development, but also benefits the transmission of the malaria parasite. Blood feeding induces massive metabolic changes that re-direct all resources towards reproduction, a state that is unsustainable over a long period of time. Here, we provide evidence that miR-276 contributes to a negative feedback loop that resets mosquito metabolism by repressing BCAA catabolism. This metabolic reset restricts mosquito reproductive investment and, thereby, benefits $P$. falciparum sporogony. Our results highlight a crucial role of mosquito reproduction in vector competence.

The fat body is the major insect storage organ, which produces the majority of hemolymph-born vitellogenic proteins and critically contributes to mosquito ovary development ${ }^{34}$. After blood feeding, high titers of AAs and 20E promote a metabolic switch in the fat body from anabolism to catabolism, resulting in a rapid release of glycogen and lipid reserves ${ }^{1,2}$. The same signals induce expression of negative regulators, including miR-276, that warrant the timely reset of the fat body metabolism ${ }^{14,15,25}$. Massive degradation of AAs in the fat body supports high energy demands of nutrient mobilization and protein synthesis for successful egg development ${ }^{8,10}$. However, high levels of AA degradation also generate a considerable amount of nitrogen waste, which may restrain the metabolic investment during the reproductive cycle ${ }^{35-37}$. Interestingly, Aedes mosquitoes lack the full repertoire of the urea cycle enzymes and rely on an alternative ammonia detoxification pathway engaging glutamine, alanine, and proline $\mathrm{e}^{32,38}$. We did not observe miR-276-driven changes in these metabolites at any tested time point. Instead, we detected increased levels of glutamate and other classic urea cycle metabolites (citrulline, ornithine, argininosuccinate, and arginine) during the late phase of the reproductive cycle. These results point to potentially important metabolic differences between Aedes and Anopheles mosquitoes and call for further targeted metabolome investigations using $\mathrm{N}^{15}$-labelled BCAAs to determine the urea cycle components in both mosquito species. The timing and nature of metabolic changes observed in this study suggest that miR-276 contributes to fine regulation of AA catabolism in the late phase of the reproductive cycle. During this phase, the fat body undergoes a reverse shift from catabolic to anabolic metabolism in order to replenish nutrient resources in preparation for the next reproductive cycle ${ }^{1,2}$. As part of this metabolic switch, miR-276 post-transcriptionally represses translation of the mRNA encoding the branched chain amino acid transferase, the catalyzer of the first step of BCAA breakdown. BCAA turnover represents a central hub that shapes the overall metabolic state and is tightly regulated ${ }^{39-42}$. In Drosophila, BCAA degradation is controlled by miR-277 ${ }^{43}$. Interestingly, the BCAA degradation pathway in Aedes shapes the bacterial load in the midgut by an as yet unknown mechanism ${ }^{44}$. Therefore, in addition to the fat body examined here, posttranscriptional regulation of BCAA metabolism by miR-276 may affect mosquito physiology at multiple levels, including the midgut microbiome. Our results show that inhibition of miR-276 prolongs high levels of BCAA catabolism and benefits female fertility. These conclusions are further supported by the observed changes in expression of the major yolk protein gene Vitellogenin $(V g)$ (Supplementary Fig. 3). As $V g$ lacks miR-276 binding sites in its $3^{\prime}$ UTR and cannot be directly regulated by this miRNA, the prolongation of the AA catabolism must be coordinated with lipid metabolism, an interesting observation which calls for further investigation. Based on these results, we propose that miR-276 contributes to a negative feedback loop that shifts the catabolic metabolism induced by AAs and 20E during the early-/ mid-phase of the female reproductive cycle to the anabolic phase. Timely attenuation of the catabolic phase is important to restrict the costly reproductive investment and prevent complete nutrient exhaustion (Fig. 5a). Metabolic costs likely play an important role in the regulation of reproductive investment as blood digestion in hematophagous insects escalates oxidative stress and nitrogen waste products $36,37,45$. Indeed, blood digestion reduces the mitochondrial activity of the flight muscles, presumably to mitigate the overall metabolic stress ${ }^{46}$. Furthermore, timely conclusion of the reproductive program is essential in preparation for the next reproductive cycle ${ }^{14,15}$. We propose that the duration of the reproductive cycle is tightly regulated by a balance between resource availability and reproductive costs.

Malaria parasites critically rely on mosquito hematophagy for sexual reproduction and transmission. Earlier studies focused on the trade-off between mosquito reproduction and Plasmodium development comparing reproductive readouts of infected versus uninfected mosquitoes ${ }^{47,48}$. However, mosquito females complete egg development before massive parasite proliferation, thereby avoiding direct competition for nutrients with Plasmodium parasites ${ }^{19,21}$. Here, we experimentally demonstrate that prolonging high levels of the AA catabolic phase by inhibition of miR-276 increases mosquito reproductive investment and a
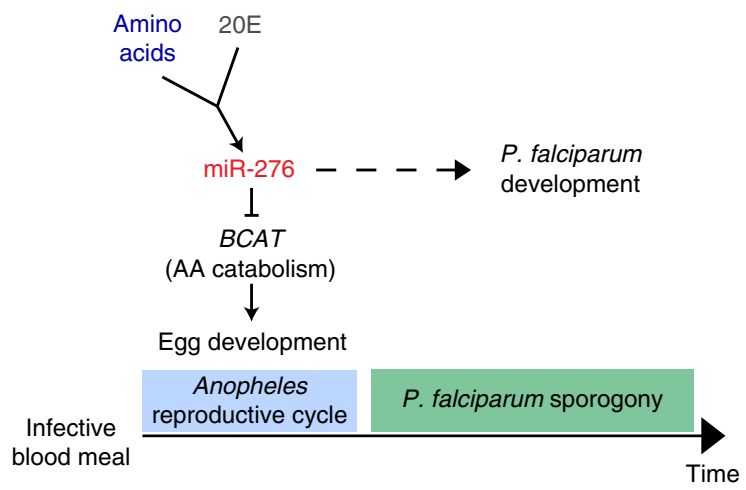

b

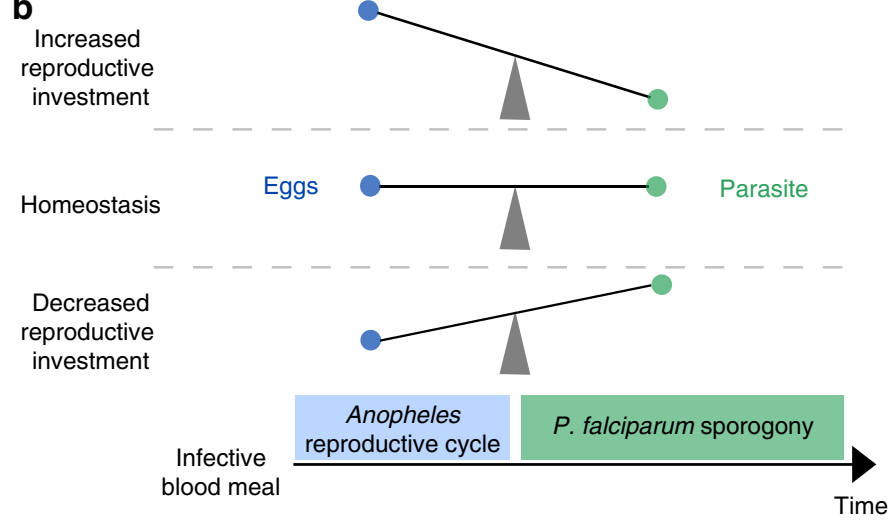

Fig. 5 Summary of miR-276 function and model of mosquito reproductive investment and $\boldsymbol{P}$. falciparum development interaction. a Amino acids ( $A$ A) and 20-hydroxyecdysone (20E) induce expression of miR-276 after blood feeding and, thereby, initiate the termination of the fat body AA catabolism by inhibiting the branched chain amino acid transferase (BCAT). This catabolism to anabolism switch in the fat body restricts mosquito investment into reproduction. P. falciparum sporogonic stages parasitize spare mosquito resources for their own development. $\mathbf{b}$ Mosquito nutritional status and the extent of the reproductive investment shape the within-vector environment which shapes $P$. falciparum sporogonic development. 
restricts the sporogonic development of $P$. falciparum. We propose that the balance between the mosquito metabolic status and reproductive investment determines Plasmodium sporogony and transmission (Fig. 5b). Importantly, variability in reproductive investment strategies could be one of the factors underlying the differences in the vector competence observed between mosquito species that are equally exposed to Plasmodium infections ${ }^{49}$.

Together with the previous reports ${ }^{19,21}$, our study describes a general mechanism by which genetic or hormonal manipulations of the mosquito metabolic program and resource allocation impinge on female fertility, within-vector Plasmodium development and within-host virulence. We propose that the mosquito metabolic status and reproductive investment are essential components of vector competence that shape malaria transmission.

\section{Methods}

Mosquitoes. Anopheles coluzzii Ngousso $\left(T E P 1{ }^{*} S 1\right)$ strain was used throughout the study. Mosquitoes were maintained at $30{ }^{\circ} \mathrm{C} 80 \%$ humidity at $12 / 12 \mathrm{~h}$ day/night cycle with a half-hour long dawn/dusk period. All mosquitoes were fed ad libitum with $10 \%$ sugar solution.

Blood meal and P. falciparum infections. Asexual cultures (parasitaemia $>2 \%$ ) of P. falciparum NF54 (kindly provided by Prof. R. Sauerwein, RUMC, The Netherlands) were harvested by centrifugation for $5 \mathrm{~min}$ at $1,500 \mathrm{rpm}$ and diluted with fresh red blood cells (RBS) (Haema) to $1 \%$ total parasitaemia at $4 \%$ hematocrit. Asexual and gametocyte cultures were incubated at $37^{\circ} \mathrm{C}$ with $3 \% \mathrm{O}_{2}$ and $4 \% \mathrm{CO}_{2}$. Gametocyte medium was changed daily for $15-16$ days on heated plates to reduce temperature drop. On day 14 after seeding, gametocytaemia was gauged by Giemsa-stained smears, and parasite exflagellation rates were evaluated by microscopy.

Mosquitoes were fed using an artificial feeder system ${ }^{50,51}$ with diluted gametocyte cultures 15-16 days after seeding at a final gametocytaemia of $0.15-0.22 \%$. The feeding system was prepared by covering the bottom of the feeders with a stretched parafilm. The feeder was heated to $37^{\circ} \mathrm{C}$ by water flow. Mosquitoes were fed for $15 \mathrm{~min}$ and unfed mosquitoes were removed. Only fully engorged females were kept for further analyses at $26^{\circ} \mathrm{C} 80 \%$ humidity.

Parasite quantification. For $P$. falciparum oocyst counts, infected mosquitoes were killed in $70 \%$ ethanol on day 11 after infection. Mosquitoes were washed twice in PBS, midguts were dissected in $1 \%$ mercurochrome in water and incubated for $10 \mathrm{~min}$ at room temperature. Oocysts were counted under a stereoscope. For oocyst size, three representative images of midgut oocysts were taken per midgut by light microscope at 40x magnification and oocysts size was gauged by Fiji Image. The sporogonic index was calculated by multiplying the median number of oocysts and the mean oocyst size per experiment.

The numbers of $P$. falciparum sporozoites in the salivary glands were evaluated 14 days post infection. At least 30 mosquitoes per condition were killed in $70 \%$ ethanol, washed with PBS and the salivary glands were dissected in RPMI medium (Gibco) 3\% bovine serum albumin (Sigma Aldrich). The salivary glands were homogenized with a glass plunger to release the sporozoites. The homogenate was filtered twice with cell strainers ( 100 and $40 \mu \mathrm{m}$ ) into glass vials and diluted at 1:10, 1:20, and 1:50. The sporozoites were counted using a hemocytometer under a light microscope. Sample sizes per experiment are provided in Supplementary Table 3.

20-hydroxyecdysone (20E) quantification. 20E levels were quantified using the 20E EIA kit (Cayman Chemical). Nine females per time point were collected into the microtubes, snap frozen and kept at $-80^{\circ} \mathrm{C}$. On the day of analysis, all samples were homogenized in $500 \mu \mathrm{l}$ methanol using steal beads and a Qiagen tissue lyser at $50 \mathrm{rpm}$ for $10 \mathrm{~min}$. Samples were centrifuged at $15,000 \mathrm{rpm}$ for $1 \mathrm{~min}$ and the supernatant $(420 \mu \mathrm{l})$ was transferred to a clean tube. The methanol was evaporated in a speedvac. The pellets were dissolved in $230 \mu \mathrm{l}$ of EIA buffer (Cayman Chemical). A 1:3 dilution series of 20E starting from $361 \mathrm{ng} / \mathrm{ml}$ served as a standard curve. Measurements were performed according to the manufacturer's instructions.

Ex vivo fat body tissue culture. Ex vivo fat body culture was performed as following: amino acid, salt, calcium and TRIS buffer stock solution was prepared according to Chung et al. ${ }^{27}$, sterile filtered and stored at $-20^{\circ} \mathrm{C}$. The basic medium was prepared without amino acids, which were replaced with an equal molarity of mannitol. 20E was dissolved in ethanol and equal amounts of ethanol were added to the medium control. Three abdominal carcasses of 3-4-day-old females were dissected and incubated with: (i) medium, (ii) $10^{-6} \mathrm{M} 20 \mathrm{E}$, (iii) amino acids (AAs); and (iv) $10^{-6} \mathrm{M} 20 \mathrm{E}$ (Sigma) and $\mathrm{AAs}^{27}$. The abdominal carcasses cultures were incubated for $6 \mathrm{~h}$ at $27^{\circ} \mathrm{C}$. RNA was isolated by RNAzol for quantification of miRNA/mRNA expression.
Sample collection for RNA expression analysis. For miRNA and mRNA expression kinetics, ten sugar-fed or blood-fed females were dissected on ice at different time points after a blood meal. The abdominal carcasses (the fat body) were immediately homogenized in RNAzol (Sigma Aldrich) and kept at $-80{ }^{\circ} \mathrm{C}$ for RNA isolation. Total RNA was isolated by RNAzol according to the manufacturer's recommendations including the purification step with 4-Bromoanizol. The total RNA yield was measured with a Qubit (Thermo Fisher Scientific).

miRNA expression analysis. miRNA expression was quantified using the miScript PCR System (Qiagen) that allows real-time PCR quantification of mRNA and miRNA expression from the same reverse transcription reaction mix. The cDNA levels were further measured by Quantitect SYBR Green PCR kit (Qiagen). Forward miR-276 primer was obtained from the miScript primer assay and reverse primer was synthesized using the sequence stated in the Supplementary Table $1^{24}$ Expression data were calculated by the relative standard curve method. Relative quantities of miRNA expression were normalized to the gene encoding the ribosomal protein S7 (RPS7).

mRNA expression analysis. For target identification, RNAs were reverse transcribed using the RevertAid H Minus First Strand cDNA synthesis kit (Thermo Fisher Scientific) and analyzed by RT-qPCR using the SYBR Green PCR mix (Thermo Fisher Scientific). Expression data was calculated using relative standard curve method. Relative quantities of miRNA expression were normalized to the gene encoding ribosomal protein S7 (RPS7). Primers sequences are listed in Supplementary Table 1 .

Computational miRNA target prediction. miRNA targets were predicted in silico using three independent algorithms: miRANDA ${ }^{29}$, RNAhybrid $^{30}$, and microTAR 31

miRNA inhibition. Antagomirs (anti-miR-276-5p and a scrambled version of the same antagomir that has no target in A. gambiae genome) were designed using the RNA module for custom single-stranded RNA synthesis (Dharmacon) as RNA antisense oligos with $2^{\prime}$-O-methylated bases, a phosphorothioate backbone at the first two and last four nucleotides and a 3 'cholesterol (Supplementary Table 2). For miRNA inhibition, mosquitoes were anesthetized with $\mathrm{CO}_{2}$ and microinjected with $207 \mathrm{nl}$ of $200 \mu \mathrm{M}$ antagomir ( $41 \mathrm{pmol} /$ female) at $12-18 \mathrm{~h}$ post eclosion using the Drummond NanoJect II (Drummond Scientific). Mosquitoes were left for four days to recover before a blood feeding or P. falciparum infection.

Egg laying and hatching assay. Female and male mosquitoes were maintained in the same cage. For fertility assays, individual females were gently transferred into single cups with egg dishes (wet Whatman paper) on day three after blood feeding and allowed to oviposit for two nights. Eggs were counted by stereoscope. For fecundity assays, females were kept together after a blood feeding and allowed to lay eggs on a common egg dish. After egg laying, the egg dish was kept in water for one day at $26^{\circ} \mathrm{C} 80 \%$ humidity. Larval hatching rates were gauged by counting the number of open egg shells (at least 100 eggs) using a stereoscope.

Dual luciferase assay. In vitro target validation was performed using Drosophila S2 cells (Invitrogen). The cells were kept in Schneider Drosophila medium supplemented with $10 \%$ heat-inactivated FBS (Gibco) and 1\% Penicillin Streptomycin (vol/vol) (Thermo Fisher Scientific) at $25^{\circ} \mathrm{C}$. Three luciferase constructs were examined: (1) a positive control containing three sites reverse complementary to miR-276 with four nucleotide linkers between each miR-276 binding site; (2) A. gambiae BCAT 3'UTR and (3) A. gambiae BCAT 3'UTR with a scrambled miR276 binding site. All constructs were separately inserted into the multiple cloning region located downstream of the Renilla translational stop codon within the psiCheck-2 vector (Promega). psiCheck-2 reporters $(100 \mathrm{ng})$ and a synthetic A. gambiae miR-276-5p miScript miRNA Mimic (100 ng, Qiagen) at a final concentration of $100 \mathrm{~nm}$ were co-transfected into Drosophila S2 cells using FuGENE HD transfection reagent (Promega). Cells transfected only with psiCheck-2 reporters were used as a "no miRNA mimic" control. Dual Luciferase Reporter Assay was performed $48 \mathrm{~h}$ post transfection using the Dual Luciferase Reporter Assay System (Promega). Firefly luciferase in the psiCheck-2 Vector was used for normalization of the Renilla luciferase expression. Measurements were made in triplicates, and transfections were repeated three times independently.

Sample preparation for untargeted metabolomics. Female mosquitoes (1-dayold) were injected with anti-miR-276-5p or scrambled antagomirs as described above. Four days later, mosquitoes were infected with P. falciparum. At 10, 38, and $48 \mathrm{hpb}$, ten females per treatment were snap-frozen in liquid nitrogen. The samples were stored at $-80^{\circ} \mathrm{C}$ until preparation. Additionally, at 38 and $48 \mathrm{hpb}$, abdominal carcasses (the fat body) of ten female mosquitoes per group were collected for miRNA target analysis by RT-qPCR.

Untargeted metabolomics was performed by the company MetaSysX GmbH, Potsdam, Germany. The sample preparation was performed according to MetaSysX standard procedure, a modified protocol from Salem et al. ${ }^{52}$. Whole 
bodies of ten snap-frozen mosquitoes were grounded into a homogenous fine powder using a tissue homogenizer. The polar metabolites and lipophilic compounds were extracted by methyl-tert-butyl-ether (MTBE)/methanol/water solvent system that separates molecules into aqueous and organic phase, respectively. After MTBE extraction, the organic phase containing lipids and lipophilic compounds was transferred to $1.5 \mathrm{ml}$ tube. The leftover of the organic phase was removed with a vacuum aspirator and $500 \mu \mathrm{l}$ of aqueous phase containing semi-polar and polar compounds was collected to a new tube. Additionally, $150 \mu \mathrm{l}$ of the polar phase were transferred to a fresh tube for subsequent derivatization and GC-MS analysis. All samples were dried down using a centrifugal evaporator and stored at $-80^{\circ} \mathrm{C}$ until LC-MS and GC-MS analysis.

Liquid chromatography mass spectrometry. For polar and lipid measurements, dried samples were resuspended in $170 \mu \mathrm{l}$ of water or acetonitrile, respectively. A $2 \mu \mathrm{l}$ injection volume was analyzed. Small molecules were separated by ultraperformance liquid chromatography (UPLC) and analyzed on an QExactive Orbitrap MS (Thermo Fisher Scientific) in positive and negative polarity.

The samples were measured with a Waters ACQUITY Reversed Phase Ultra Performance Liquid Chromatography (RP-UPLC) coupled to a Thermo-Fisher QExactive mass spectrometer. $\mathrm{C}_{8}(100 \mathrm{~mm} \times 2.1 \mathrm{~mm} \times 1.7 \mu \mathrm{m}$ particles; Waters $)$ and HSS T3 $\mathrm{C}_{18}(100 \mathrm{~mm} \times 2.1 \mathrm{~mm} \times 1.8 \mu \mathrm{m}$ particles; Waters $)$ columns were used for the lipophilic and the hydrophilic measurements, respectively. A $15 \mathrm{~min}$ gradient was used for separation of polar and lipophilic compounds. The mobile phases for separation of polar and semi-polar compounds were $0.1 \%$ formic acid in $\mathrm{H}_{2} \mathrm{O}$ (buffer A) and $0.1 \%$ formic acid in acetonitrile (buffer B). The chromatographic separation of these analytes was performed in the following conditions: A $99 \%$ initial to $1 \mathrm{~min}$, A $99 \%$ to A $60 \%$ from 0 to $11 \mathrm{~min}, \mathrm{~A} 60 \%$ to A $30 \%$ from 11 to $13 \mathrm{~min}$ and $\mathrm{A} 30 \%$ to A $1 \%$ from 13 to $15 \mathrm{~min}$. The following mobile phases were used for lipids and lipophilic metabolites separation: $1 \%$ of $1 \mathrm{~m}$ $\mathrm{NH}_{4} \mathrm{Ac}$ in $0.1 \%$ acetic acid (buffer A) and acetonitrile:isopropanol (7:3) containing $1 \%$ of $1 \mathrm{M} \mathrm{NH}_{4} \mathrm{Ac}$ in $0.1 \%$ acetic acid (buffer B). The separation of lipids and lipophilic compounds was performed with a step gradient from $45 \%$ initial to $1 \mathrm{~min}, 45$ to $25 \% \mathrm{~A}$ in $4 \mathrm{~min}, 25$ to $11 \% \mathrm{~A}$ in $11 \mathrm{~min}$ and 11 to $0 \% \mathrm{~A}$ in $15 \mathrm{~min}$. Chromatograms were recorded in Full Scan MS mode (Mass Range [100-1,500]).

All mass spectra were acquired in positive and negative mode with the following settings of the instrument: Heated electrospray ionization (HESI) was used, spray voltage was $3.5 \mathrm{kV}$, capillary temperature $-275^{\circ} \mathrm{C}$, sheath gas flow rate -60 units, mass resolving power 70,000, $3 \times 10^{6}$ target value (AGC) and maximal fill time of $200 \mathrm{~ms}$. For lipid annotation, all samples were pooled and measured four times in LC-MS/MS mode. The fragmentation was performed in data-dependent MS/MS mode using higher-energy collisional dissociation (HCD). Three most intense ions were selected for fragmentation per cycle using a normalized collision energy of 25 . The full scan spectra were acquired in the $100-1,500 \mathrm{~m} / \mathrm{z}$ range at a mass resolution of 35,000 with an AGC of $10^{5}$ ions with maximal fill time of $100 \mathrm{~ms}$. The target values for data dependent MS/MS scans were set to $5 \times 10^{4}$ ion with a maximal fill time of $50 \mathrm{~ms}$ and an isolation window of $1 \mathrm{Th}$. The MS/MS ions were measured at a resolution 17,500 and the dynamic exclusion was set to $3 \mathrm{~s}$. Thermo Excalibur was used for the data acquisition.

An in-house metaSysX database of chemical compounds was used to match the features detected in the LC-MS polar and non-polar platform. The metaSysX database contains the mass-to-charge ratio and the retention time information of reference compounds measured at the same chromatographic and spectrometric condition as samples measurements. The matching criteria for the polar platform were $5 \mathrm{ppm}$ and $0.095 \mathrm{~min}$ deviation from the reference compounds mass-tocharge-ratio and retention time respectively and for lipid annotation $5 \mathrm{ppm}$ and the range $(-0.095 ; 0.255 \mathrm{~min})$ time deviation. Coeluting compounds with the same ion mass were kept as unsolved annotation. Lipid annotation was additionally confirmed by MS/MS generated fragments. A metaSysX-developed R-based algorithm uses information from fragments, neutral losses presence and precursor masses information detected in both ionisations' modes. This information is combined with the information obtained from the database search to give an additional level of confidence. The lipid database was created based on the precursor ion mass, fragmentation spectrum, and elution pattern ${ }^{53}$. The elution patterns of annotated lipids are used to provide an additional level of confidence for lipid annotation.

Gas chromatography mass spectrometry. Dried samples were suspended in methoxy-hydrochloride/pyridine solution and incubated at $30^{\circ} \mathrm{C}$ for $90 \mathrm{~min}$ followed by derivatization with $\mathrm{N}$-methyl-N-trimethylsilyltrifluoroacetamide (MSTFA) at $37^{\circ} \mathrm{C}$ for $30 \mathrm{~min}^{54}$. The samples were measured on an Agilent Technologies GC coupled to a Leco Pegasus HT mass spectrometer which consists of an EI ionization source and a TOF mass analyzer. Column: $30 \mathrm{~m}$ DB35; starting temp: $85^{\circ} \mathrm{C}$ for $2 \mathrm{~min}$; gradient: $15^{\circ} \mathrm{C}$ per min up to $360^{\circ} \mathrm{C}$

NetCDF files that were exported from the Leco Pegasus software were imported into the " $R$ " Bioconductor package TargetSearch ${ }^{55}$ to transform retention time to retention index (RI), to align the chromatograms, to extract the peaks, and to annotate them by comparing the spectra and the RI to the Fiehn Library and to a user created library. Annotation of peaks was manually confirmed in Leco Pegasus. Analytes were quantified using a unique mass. Metabolites with a RT and a mass spectrum that did not have a match in the database were labelled as unknown.
Data filtering and normalization. The GC- and LC-MS datasets were normalized to the sample median intensity. For heatmap visualization, missing data were replaced with the smallest value of the detected metabolite. Furthermore, the remaining dataset was subjected to interquartile range filtering. The data was then $\log 2$ transformed and scaled by mean-centering and by division by the square root of each feature (pareto scaling). The raw GC-MS and LC-MS dataset is attached as Supplementary material (Supplementary Data 3).

Reporting summary. Further information on research design is available in the Nature Research Reporting Summary linked to this article.

\section{Data availability}

The polar and lipid LC-MS data in positive and negative polarity as well as GC-MS data that support the findings of this study have been deposited in the MetaboLights repository with the accession code MTBLS1282. The paper is available as preprint on the bioRxiv server [https://doi.org/10.1101/548784]. Source data for Figs. 1, 2, 4 are provided in the Source Data file.

Received: 25 February 2019; Accepted: 14 November 2019; Published online: 10 December 2019

\section{References}

1. Hou, Y. et al. Temporal coordination of carbohydrate metabolism during mosquito reproduction. PLoS Genet. 11, e1005309 (2015).

2. Wang, $X$. et al. Hormone and receptor interplay in the regulation of mosquito lipid metabolism. Proc. Natl Acad. Sci. USA 114, E2709-E2718 (2017).

3. Ziegler, R. \& Ibrahim, M. M. Formation of lipid reserves in fat body and eggs of the yellow fever mosquito, Aedes aegypti. J. Insect Physiol. 47, 623-627 (2001).

4. Briegel, H., Hefti, M. \& DiMarco, E. Lipid metabolism during sequential gonotrophic cycles in large and small female Aedes aegypti. J. Insect Physiol. 48, 547-554 (2002)

5. Carpenter, V. K. et al. SLC7 amino acid transporters of the yellow fever mosquito Aedes aegypti and their role in fat body TOR signaling and reproduction. J. insect Physiol. 58, 513-522 (2012).

6. Hansen, I. A., Attardo, G. M., Roy, S. G. \& Raikhel, A. S. Target of rapamycindependent activation of S6 kinase is a central step in the transduction of nutritional signals during egg development in a mosquito. J. Biol. Chem. 280, 20565-20572 (2005)

7. Roy, S. et al. Regulation of Gene Expression Patterns in Mosquito Reproduction. PLoS Genet. 11, e1005450 (2015).

8. Zhou, G. et al. Metabolic fate of [14C]-labeled meal protein amino acids in Aedes aegypti mosquitoes. J. Insect Physiol. 50, 337-349 (2004).

9. Zhou, G., Pennington, J. E. \& Wells, M. A. Utilization of pre-existing energy stores of female Aedes aegypti mosquitoes during the first gonotrophic cycle. Insect Biochem. Mol. Biol. 34, 919-925 (2004).

10. Fuchs, S., Behrends, V., Bundy, J. G., Crisanti, A. \& Nolan, T. Phenylalanine metabolism regulates reproduction and parasite melanization in the malaria mosquito. PLoS One 9, e84865 (2014).

11. Gulia-Nuss, M., Robertson, A. E., Brown, M. R., Strand, M. R. \& Pankratz, M. Insulin-Like Peptides and the Target of Rapamycin Pathway Coordinately Regulate Blood Digestion and Egg Maturation in the Mosquito Aedes aegypti. PLoS One 6, e20401 (2011).

12. Hansen, I. A., Attardo, G. M., Park, J.-H., Peng, Q. \& Raikhel, A. S. Target of rapamycin-mediated amino acid signaling in mosquito anautogeny. Proc. Natl Acad. Sci. USA 101, 10626-10631 (2004).

13. Rono, M. K., Whitten, M. M. A., Oulad-Abdelghani, M., Levashina, E. A. \& Marois, E. The major yolk protein vitellogenin interferes with the antiplasmodium response in the malaria mosquito Anopheles gambiae. PLoS Biol. 8, e1000434 (2010).

14. Mane-Padros, D., Cruz, J., Cheng, A. \& Raikhel, A. S. A critical role of the nuclear receptor HR3 in regulation of gonadotrophic cycles of the mosquito Aedes aegypti. PLoS One 7, e45019 (2012).

15. Bryant, B. \& Raikhel, A. S. Programmed autophagy in the fat body of Aedes aegypti is required to maintain egg maturation cycles. PLoS One 6, e25502 (2011).

16. Stone, W. J. R. et al. The relevance and applicability of oocyst prevalence as a read-out for mosquito feeding assays. Sci. Rep. 3, 272 (2013).

17. Takken, W. et al. Larval nutrition differentially affects adult fitness and Plasmodium development in the malaria vectors Anopheles gambiae and Anopheles stephensi. Parasites vectors 6, 345 (2013).

18. Moller-Jacobs, L. L., Murdock, C. C. \& Thomas, M. B. Capacity of mosquitoes to transmit malaria depends on larval environment. Parasit. Vectors 7, 593 (2014). 
19. Costa, G. et al. Non-competitive resource exploitation within mosquito shapes within-host malaria infectivity and virulence. Nat. Commun. 9, 3474 (2018).

20. Slavic, K. et al. Use of a selective inhibitor to define the chemotherapeutic potential of the plasmodial hexose transporter in different stages of the parasite's life cycle. Antimicrob. Agents Chemother. 55, 2824-2830 (2011).

21. Werling, K. et al. Steroid hormone function controls non-competitive Plasmodium development in Anopheles. Cell 177, 315-325.e14 (2019)

22. Liu, S., Lucas, K. J., Roy, S., Ha, J. \& Raikhel, A. S. Mosquito-specific microRNA-1174 targets serine hydroxymethyltransferase to control key functions in the gut. Proc. Natl Acad. Sci. USA 111, 14460-14465 (2014).

23. Lucas, K. J., Zhao, B., Roy, S., Gervaise, A. L. \& Raikhel, A. S. Mosquitospecific microRNA-1890 targets the juvenile hormone-regulated serine protease JHA15 in the female mosquito gut. RNA Biol. 12, 1383-1390 (2015).

24. Bryant, B., Macdonald, W. \& Raikhel, A. S. microRNA miR-275 is indispensable for blood digestion and egg development in the mosquito Aedes aegypti. Proc. Natl Acad. Sci. USA 107, 22391-22398 (2010).

25. Lampe, L. \& Levashina, E. A. MicroRNA tissue atlas of the malaria mosquito Anopheles gambiae. G3 (Bethesda) 8, 185-193 (2018).

26. Dennison, N. J., BenMarzouk-Hidalgo, O. J. \& Dimopoulos, G. MicroRNAregulation of Anopheles gambiae immunity to Plasmodium falciparum infection and midgut microbiota. Deve. Comp. Immunol. 49, 170-178 (2015).

27. Chung, H.-N. et al. Fat Body Organ Culture System in Aedes Aegypti, a Vector of Zika Virus. J. https://doi.org/10.3791/55508 (2017).

28. Deitsch, K. W., Chen, J.-S. \& Raikhel, A. S. Indirect control of yolk protein genes by 20-hydroxyecdysone in the fat body of the mosquito, Aedes aegypti. Insect Biochem. Mol. Biol. 25, 449-454 (1995).

29. John, B. et al. Human MicroRNA Targets. PLoS Biol. 2, e363 (2004).

30. Kruger, J. \& Rehmsmeier, M. RNAhybrid: microRNA target prediction easy, fast and flexible. Nucleic Acids Res. 34, W451-W454 (2006).

31. Thadani, R. \& Tammi, M. T. MicroTar: predicting microRNA targets from RNA duplexes. BMC Bioinforma. 7, S20 (2006).

32. Scaraffia, P. Y., Zhang, Q., Wysocki, V. H., Isoe, J. \& Wells, M. A. Analysis of whole body ammonia metabolism in Aedes aegypti using [15N]-labeled compounds and mass spectrometry. Insect Biochem. Mol. Biol. 36, 614-622 (2006).

33. Nsango, S. E. et al. AP-1/Fos-TGase2 axis mediates wounding-induced Plasmodium falciparum killing in Anopheles gambiae. J. Biol. Chem. 288, 16145-16154 (2013).

34. Arrese, E. L. \& Soulages, J. L. Insect fat body: energy, metabolism, and regulation. Annu. Rev. Entomol. 55, 207-225 (2010).

35. Isoe, J. \& Scaraffia, P. Y. Urea synthesis and excretion in Aedes aegypti mosquitoes are regulated by a unique cross-talk mechanism. PLoS One 8 e65393 (2013).

36. Mazzalupo, S., Isoe, J., Belloni, V. \& Scaraffia, P. Y. Effective disposal of nitrogen waste in blood-fed Aedes aegypti mosquitoes requires alanine aminotransferase. FASEB J. 30, 111-120 (2016).

37. Isoe, J. et al. Xanthine dehydrogenase-1 silencing in Aedes aegypti mosquitoes promotes a blood feeding-induced adulticidal activity. FASEB J. 31, 2276-2286 (2017).

38. Scaraffia, P. Y., Zhang, Q., Thorson, K., Wysocki, V. H. \& Miesfeld, R. L. Differential ammonia metabolism in Aedes aegypti fat body and midgut tissues. J. Insect Physiol. 56, 1040-1049 (2010).

39. She, P. et al. Disruption of BCATm in mice leads to increased energy expenditure associated with the activation of a futile protein turnover cycle. Cell Metab. 6, 181-194 (2007).

40. Li, T. et al. Defective branched-chain amino acid catabolism disrupts glucose metabolism and sensitizes the heart to ischemia-reperfusion injury. Cell Metab. 25, 374-385 (2017)

41. Zhang, S., Zeng, X., Ren, M., Mao, X. \& Qiao, S. Novel metabolic and physiological functions of branched chain amino acids: a review. J. Anim. Sci. Biotechnol. 8, 10 (2017).

42. Yoon, M.-S. The emerging role of branched-chain amino acids in insulin resistance and metabolism. Nutrients 8, pii: E405(2016)

43. Esslinger, S. M. et al. Drosophila miR-277 controls branched-chain amino acid catabolism and affects lifespan. RNA Biol. 10, 1042-1056 (2013).

44. Short, S. M., Mongodin, E. F., MacLeod, H. J., Talyuli, O. A. C. \& Dimopoulos, G. Amino acid metabolic signaling influences Aedes aegypti midgut microbiome variability. PLoS Negl. Trop. Dis. 11, e0005677 (2017).

45. Magalhaes, T., Brackney, D. E., Beier, J. C. \& Foy, B. D. Silencing an Anopheles gambiae catalase and sulfhydryl oxidase increases mosquito mortality after a blood meal. Arch. Insect Biochem. Physiol. 68, 134-143 (2008).

46. Gonçalves, R. L. S. et al. Blood-feeding induces reversible functional changes in flight muscle mitochondria of Aedes aegypti mosquito. PloS One 4, e7854 (2009).
47. Jahan, N. \& Hurd, H. Effect of Plasmodium yoelii nigeriensis (Haemosporidia Plasmodiidae) on Anopheles stephensi (Diptera: Culicidae) vitellogenesis. J. Med. Entomol. 35, 956-961 (1998).

48. Ahmed, A. M., Maingon, R., Romans, P. \& Hurd, H. Effects of malaria infection on vitellogenesis in Anopheles gambiae during two gonotrophic cycles. Insect Mol. Biol. 10, 347-356 (2001).

49. Briegel, H. Mosquito reproduction: Incomplete utilization of the blood meal protein for oögenesis. J. Insect Physiol. 31, 15-21 (1985).

50. Lensen, A. et al. Infectivity of cultured Plasmodium falciparum gametocytes to mosquitoes. Exp. Parasitol. 91, 101-103 (1999).

51. Lensen, A. et al. Measurement by membrane feeding of reduction in Plasmodium falciparum transmission induced by endemic sera. Trans. R. Soc. Trop. Med. Hyg. 90, 20-22 (1996).

52. Salem, M. A., Jüppner, J., Bajdzienko, K. \& Giavalisco, P. Protocol: a fast, comprehensive and reproducible one-step extraction method for the rapid preparation of polar and semi-polar metabolites, lipids, proteins, starch and cell wall polymers from a single sample. Plant Methods 12, 45 (2016).

53. Bromke, M. A. et al. Liquid chromatography high-resolution mass spectrometry for fatty acid profiling. Plant J. 81, 529-536 (2015).

54. Lisec, J., Schauer, N., Kopka, J., Willmitzer, L. \& Fernie, A. R. Gas chromatography mass spectrometry-based metabolite profiling in plants. Nat. Protoc. 1, 387-396 (2006).

55. Cuadros-Inostroza, Á. et al. TargetSearch-a Bioconductor package for the efficient preprocessing of GC-MS metabolite profiling data. BMC Bioinforma. 10, 428 (2009).

\section{Acknowledgements}

The authors wish to thank Liane Spohr for mosquito breeding, Manuela Andres and Daniel Eyermann for P. falciparum cultures and Cornelia Kreschel for sporozoite isolation and counting. Finally, we are grateful to all members of the Vector Biology Unit for fruitful discussions and to Dr. Guilia Costa, Dr. Paola Carrillo-Bustamante, and Clare Newell for constructive comments on the manuscript.

\section{Author contributions}

L.L. and E.A.L. conceived the study and designed the experiments. L.L. and M.J. performed experiments and analyzed the data. S.K. provided expertise on the untargeted metabolomics experiments. L.L. and E.A.L. wrote the paper.

\section{Competing interest}

The authors declare no competing interest.

\section{Additional information}

Supplementary information is available for this paper at https://doi.org/10.1038/s41467 019-13627-y.

Correspondence and requests for materials should be addressed to E.A.L.

Peer review information Nature Communications thanks Jianhong Ching and the other, anonymous, reviewer(s) for their contribution to the peer review of this work. Peer reviewer reports are available.

Reprints and permission information is available at http://www.nature.com/reprints

Publisher's note Springer Nature remains neutral with regard to jurisdictional claims in published maps and institutional affiliations.

Open Access This article is licensed under a Creative Commons Attribution 4.0 International License, which permits use, sharing, adaptation, distribution and reproduction in any medium or format, as long as you give appropriate credit to the original author(s) and the source, provide a link to the Creative Commons license, and indicate if changes were made. The images or other third party material in this article are included in the article's Creative Commons license, unless indicated otherwise in a credit line to the material. If material is not included in the article's Creative Commons license and your intended use is not permitted by statutory regulation or exceeds the permitted use, you will need to obtain permission directly from the copyright holder. To view a copy of this license, visit http://creativecommons.org/ licenses/by/4.0/

(C) The Author(s) 2019 